Ann. Génét. Sél. anim., I973, 5 (3), 313-32I.

\title{
EFFETS DU GÈNE DE NANISME $d w$ SUR LA PRODUCTION DE SPERMATOZOÏDES CHEZ LE COQ EN CROISSANCE
}

\author{
M. de REVIERS et M.-J. PETITJEAN* \\ avec la collaboration technique de J.-C. Folmer, Lucette BÉNARd et J.-P. BRILlard \\ Station de Recherches avicoles, \\ Centre de Recherches de Tours, I. N.R. A., \\ Nouzilly, B. P. 1,37380 Monnaie \\ * Station expérimentale d'Aviculture de Magneraud, I. N.R. A., \\ B. P. 52, 17700 Surgères
}

RÉSUMÉ

De I $_{4}$ à 28 semaines d'âge, la quantité de spermatozoïdes par éjaculat est un peu plus faible chez les coqs $d w d w$ (phénotype nain) que chez les coqs Dwdw (phénotype normal). A l'âge adulte, cette différence est de $25 \mathrm{p}$. Ioo environ, bien que le poids testiculaire des coqs $d w d w$ soit beaucoup plus faible $(\mathrm{I} 0,8 \pm 0,5 \mathrm{~g})$ que celui des coqs $D w d w(25,2 \pm \mathrm{I}, \mathrm{I} \mathrm{g})$. On constate que le gène dw déprime plus fortement le développement testiculaire que le développement corporel. Mais la motilité des spermatozoïdes éjaculés est plus élevée chez les coqs dwdw que chez les coqs $D w d w$.

Dans les deux lots de coqs, la motilité des premiers spermatozoïdes produits est très faible ; elle augmente progressivement de $\mathrm{I}_{4}$ à $\mathrm{I} 8$ semaines d'âge ; en outre, la proportion de spermatozoïdes mobiles augmente fortement lorsque les collectes de sperme deviennent plus fréquentes.

\section{INTRODUCTION}

Le gène de nanisme $d w$, décrit pour la première fois chez le poulet par HưT en I949, a fait 1'objet de nombreux travaux, excepté en ce qui concerne ses effets éventuels sur la fertilité des coqs.

Dans le présent travail, nous comparons l'évolution quantitative et qualitative des éjaculats récoltés pendant la période qui s'étend depuis l'apparition des premiers spermatozoïdes jusqu'à 1'âge de 28 semaines chez des coqs $d w d w$ (phénotype nain) 
d'une part, chez des coqs $D w d w$ (phénotype normal) d'autre part. De même, nous comparons leurs caractéristiques testiculaires à l'âge de 28 semaines.

Une partie des résultats rapportés vient d'être publiée par ailleurs (PETITJEAN et de REVIERS, I973).

\section{MATÉRIEL E'T MÉTHODES}

Ce travail porte sur $5^{\mathrm{r}}$ coqs, dont $27 d w d w$ (phénotype nain) et 24 Dwdw (phénotype normal), issus d'une population obtenue par RICARD (I970). Ces $5 \mathrm{I}$ coqs comprenaient $\mathrm{I}_{4}$ couples de frères, un frère de chaque couple étant $d w d w$, l'autre Dwdw.

Ces coqs ont d'abord été élevés au sol, puis placés dans les cages individuelles d'une batterie à partir de l'âge de I4 semaines. La température du local d'élevage a été maintenue entre I 8 et $22^{\circ} \mathrm{C}$. Les coqs ont reçu l'éclairement naturel, c'est-à-dire des jours de longueur croissante pendant toute la durée de l'expérience.

\section{A. - Récolte et caractère des éjaculats}

Tous les éjaculats ont été récoltés le matin (méthode de Burrows et Quins, 1937). La fréquence de collecte était de 2 par semaine (lundi et jeudi) entre ${ }_{4} 4$ et 22 semaines d'âge, puis I par jour (dimanche excepté) après l'âge de 22 semaines. Chaque éjaculat a été mélangé à un volume égal de dilueur ( ${ }^{1}$ ), puis refroidi et conservé pendant environ 5 heures à $+4^{\circ} \mathrm{C}$. Les mesures effectuées sur ces éjaculats ont été les suivantes :

- volume (déterminé à l'aide d'une pipette) ;

- concentration en spermatozoïdes (densité optique à $630 \mathrm{~m} \mu$, le sperme étant dilué à $\mathrm{I}, 5 \mathrm{p}$. Ioo dans du citrate trisodique à 3,6 p. Ioo) ;

- proportion de spermatozoides mobiles, mesurée à l'aide d'un appareillage électronique mis au point par Petitjean).

\section{B. - Caractéristiques des testicules}

Tous les coqs ont été abattus le même jour à l'âge de 28 semaines; leurs testicules ont été prélevés et pesés individuellement; des broyats de chacun d'eux ont été réalisés suivant une technique exposée par ailleurs (de REVIERs, I972 a) afin de déterminer par comptages hématimétriques :

- le nombre testiculaire, ou nombre total $\mathrm{N}_{t}$ de spermatides allongées et de spermatozoïdes contenus dans chaque testicule ;

- le coefficient d'activité testiculaire, rapport du nombre au poids testiculaire.

Ces deux paramètres ont été définis chez le Bélier (OrTavant, I958) ; leur signification chez le Coq a été discutée par de REviers, I972 $b$.

Étant donné le grand nombre de comptages à effectuer, nous avons dû conserver les broyats testiculaires pendant 24 à $72 \mathrm{~h}$ (dilueur : saccharose $0,25 \mathrm{M}$; température : $4^{\circ} \mathrm{C}$ ). Dans ces conditions, il n'y a pas de différences significatives $(\mathrm{F}<\mathrm{I})$ entre les résultats des comptages successifs des Io mêmes échantillons (Io grilles d'hématimètre par échantillon) après $24,4^{8}$ ou $72 \mathrm{~h}$ de conservation.

\section{RÉSULTATS}

\section{A. - Caractères comparés des éjaculats}

I. L'âge au premier éjaculat est un peu plus tardif chez les coqs nains (I52 士20 j) que chez les coqs $D w d w$ ( I $42 \pm 34 \mathrm{j}$ ) mais cette différence n'est pas significative au seuil 5 p. Ioo.

(1) Dilavia, I. N. R. A., à base de glutamate de Na, mis au point par Petitjean, commercialisé, par Virbac S. A. 06 - Nice. 
2. Pour un âge donné, le nombre de massages suivis d'éjaculation (exprimé en p. roo) est approximativement le même dans les 2 lots de coqs (fig. I). Ce nombre augmente rapidement en fonction de l'âge ; il atteint le niveau Ioo après la $23^{e}$ semaine et s'y maintient ensuite.

3. Le nombre total de spermatozoïdes par éjaculat, estimé à l'aide du produit de leur densité optique par leur volume, est presque toujours plus faible chez les coqs $d w d w$ que chez les coqs $D w d w$ (fig. 2 ). Cette différence, de l'ordre de 25 p. Ioo après

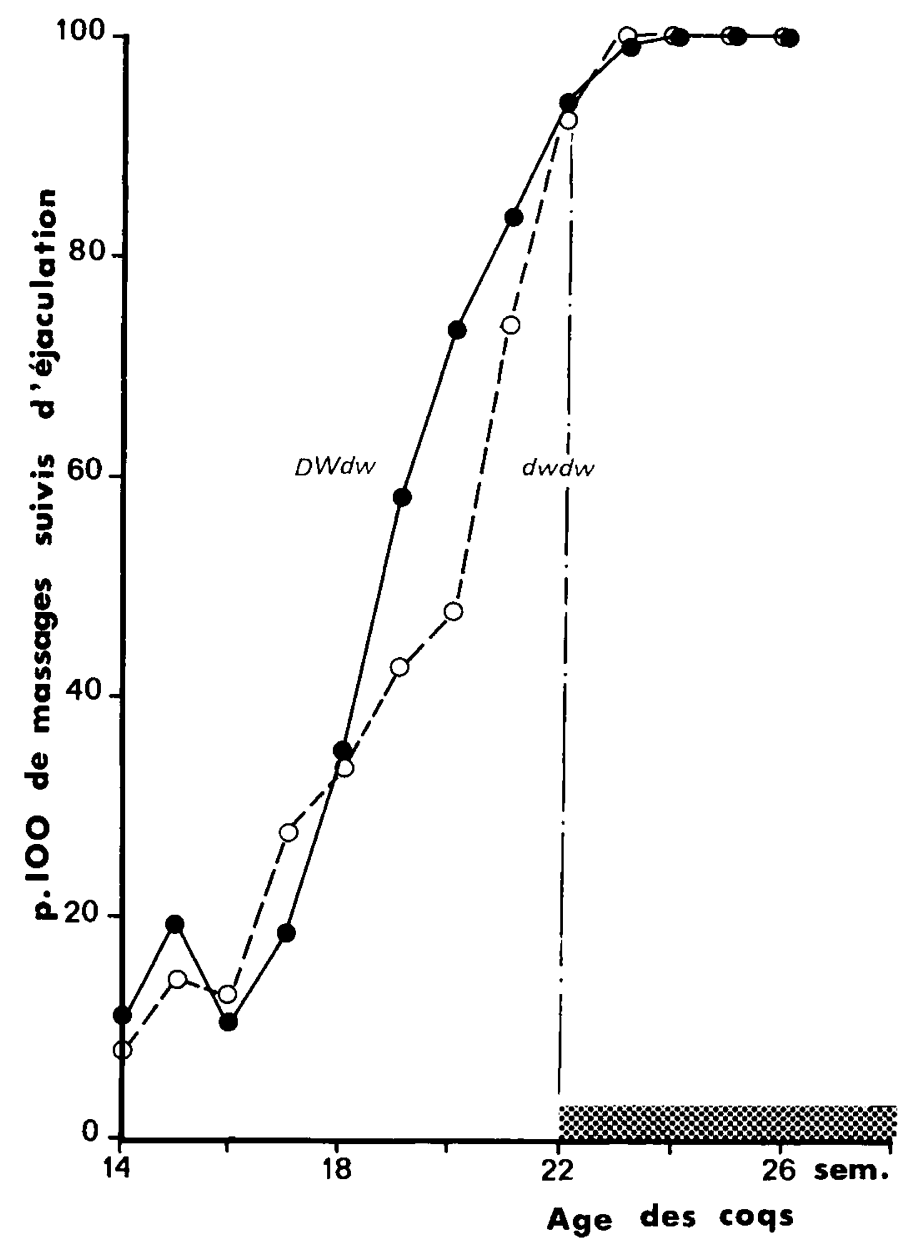

FIG. I. - Évolution comparée en fonction de l'âge du nombre des massages suivis d'éjaculation (exprimé en p.Ioo) chez les coqs Dwdw et chez les cogs dwdw

Comme dans les figures 2, 3 et 4, la zone grisée sur l'axe des abscisses correspond à la période pendant laquelle les collectes ont été effectuées $\mathrm{I}$ fois par jour, dimanches exceptés

l'âge de 23 semaines, s'explique par le plus faible volume des éjaculats des coqs nains car la densité optique des éjaculats est la même pour les deux lots de coqs (fig. 3).

Le nombre total de spermatozoïdes par éjaculat augmente très fortement en fonction de l'âge dans les deux lots jusqu'à la I9 ${ }^{\text {e }}$ semaine : de I9 à 22 semaines cette 


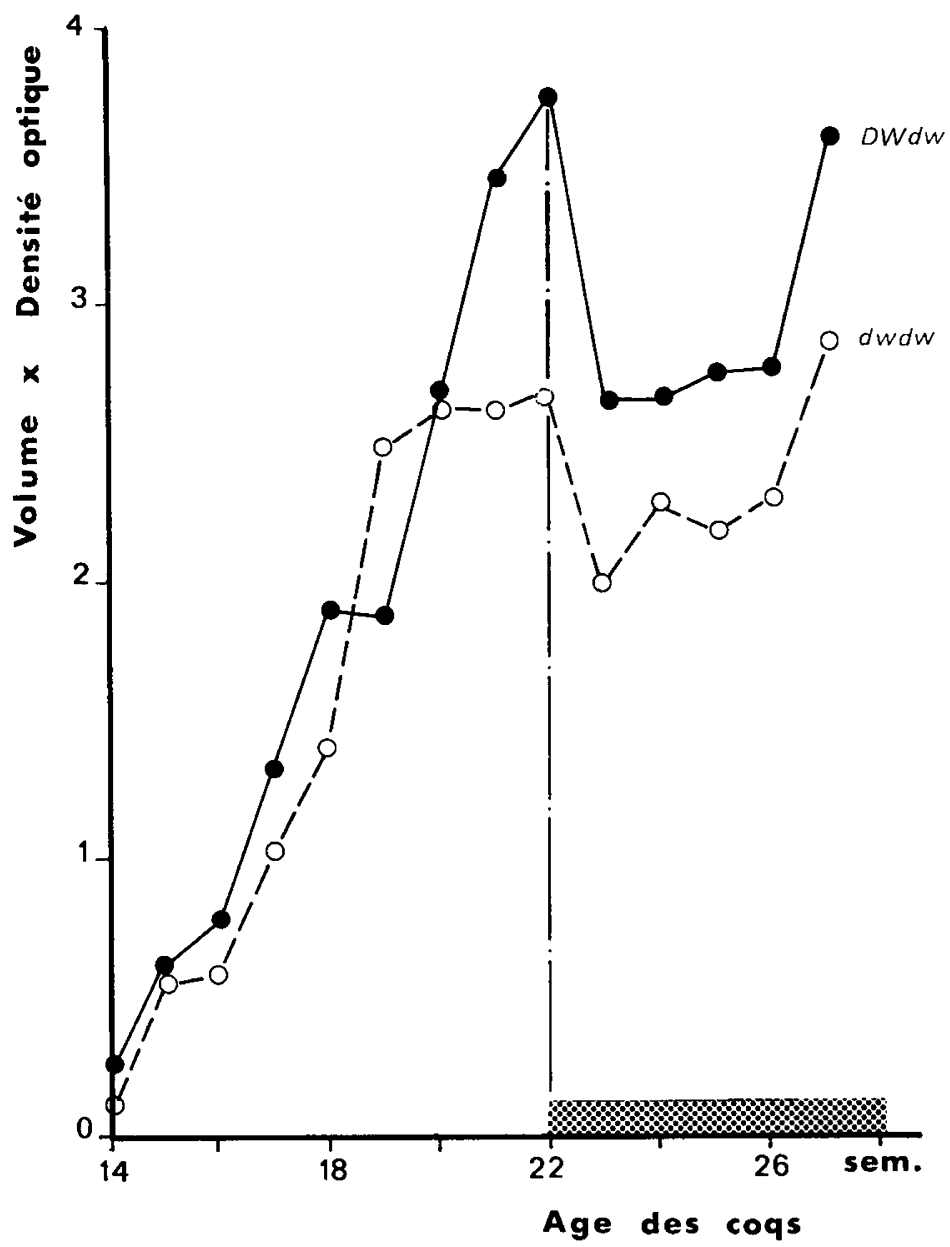

FIG. 2. - Évolution comparée en fonction de l'áge du nombre de spermatozoüdes par éjaculat (volume $x$ densité optique), chez les coqs Dwdw et chez les coqs dwdw

Cette courbe met simultanément en évidence l'effet de l'âge des coqs, du gène dw et de la fréquence des collectes 


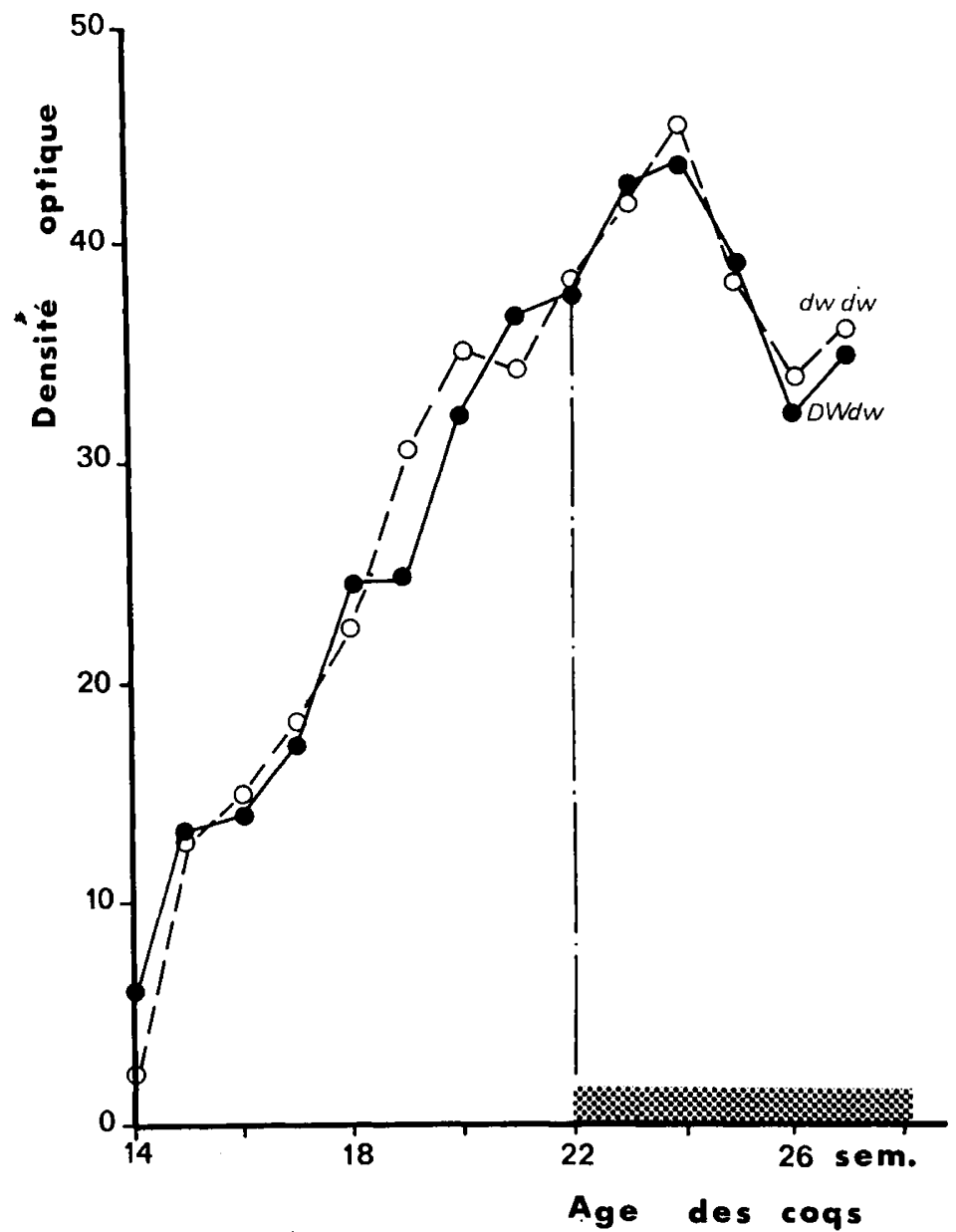

FIG. 3. - Évolution comparée en fonction de l'âge de la densité optique des éjaculats (exprimée en p. I0o), chez les coqs dwdw et chez les coqs Dwdw

Si le gène $d w$ paraît être ici sans effet, il n'en est pas de même pour l'âge, dont l'effet est très important, et pour la fréquence des collectes dont l'effet est notable 
augmentation ne se poursuit que chez les coqs $D w d w$; enfin après 23 semaines on observe une diminution brutale du nombre de spermatozoïdes par éjaculat dans les deux lots ; elle coïncide avec le changement de la fréquence des collectes. Que ce soit

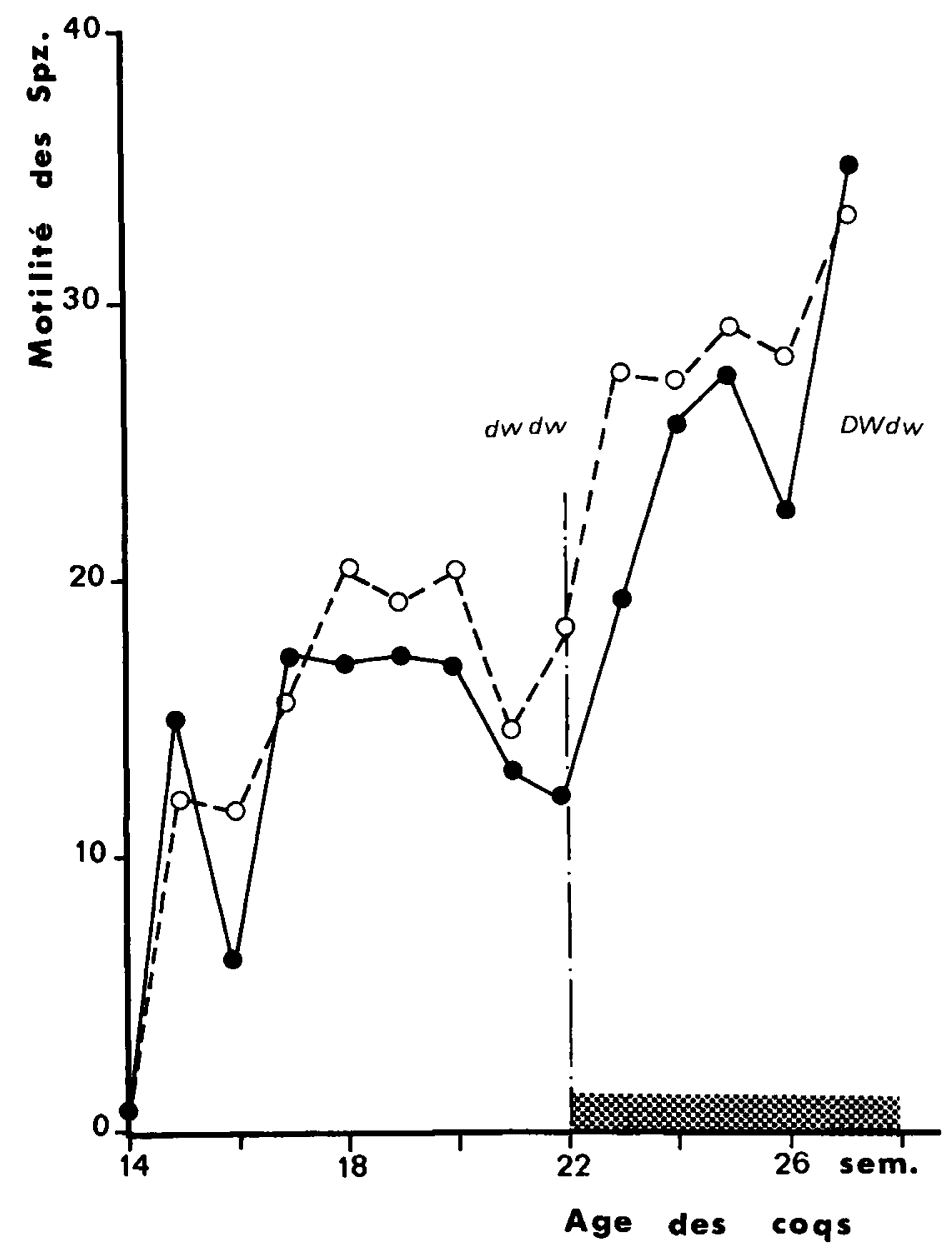

FIG. 4. - Evolution comparée en fonction de l'âge de la proportion de spermatozoüdes mobiles chez les cogs DWdw et chez les coqs dwdw.

Il apparaît que la motilité des spermatozoĩdes, très faible dans les premiers éjaculats, augmente ensuite fortement en fonction de l'âge et de la fréquence de collecte et qu'elle est en général plus élevée chez les coqs dwdw que chez les coqs DWdw.

avant l'âge de I9 semaines ou après celui de 22 semaines, nous constatons que les variations du nombre de spermatozoïdes par éjaculat proviennent de variations simultanées du volume de ces éjaculats et de leur densité optique.

4. La proportion de spermatozoides mobiles, légèrement plus élevée chez les coqs nains que chez les coqs Dwdw, augmente elle aussi en fonction de l'âge jusqu'à la I $8^{\mathrm{e}}$ semaine puis n'évolue plus de manière très notable jusqu'au changement de la fréquence des collectes; à ce moment elle augmente à nouveau très fortement (fig. 4). 


\section{B. - Caractères comparés des testicules (tabl. I)}

Chez les coqs nains, le nombre testiculaire moyen n'atteint que $43 \mathrm{p}$. Ioo de la valeur observée chez les coqs $D w d w$; il en est de même pour le poids testiculaire alors que les coefficients d'activité testiculaire sont à peu près les mêmes dans les deux lots

TABLEAU I

Comparaison des caractères testiculaires chez les coqs nains (dwdw) et normaux (Dwdw) à l'âge de 28 semaines

\begin{tabular}{|c|c|c|c|}
\hline & $d w d w$ & Dwdw & Signification \\
\hline Poids testiculaire moyen $(\mathrm{g})$ & $10,8 \pm 0,48$ & $25,2 \pm 1,12$ & $1 \%$ \\
\hline CAT (en millions/g) & $396 \pm 14$ & $383 \pm 9,6$ & NS \\
\hline Nombre testiculaire (en milliards) & $4,26 \pm 0,23$ & $9,84 \pm 0,53$ & $1 \%$ \\
\hline
\end{tabular}

$\mathrm{CAT}=$ Coefficient d'Activité testiculaire.

de coqs. La quantité de spermatozoïdes récoltée ne reflète donc pas la quantité produite par les testicules, contrairement à ce que l'un de nous a pu observer dans plusieurs expériences (de REviERS, I972 $b$ et données non publiées).

Dans chacun des deux lots, les poids et les nombres testiculaires individuels sont en corrélation élevée (0,74 chez les coqs nains et $0,87 \mathrm{chez}$ les coqs $D w d w)$.

Enfin nous observons une corrélation de 0,65 entre les poids testiculaires des coqs frères ; la corrélation observée entre ces mêmes coqs pour les nombres testiculaires est trop faible pour être significative, même au seuil Io p. Ioo ( $r=0,40$, I2 d.1.).

\section{DISCUSSION}

Nous constatons d'importantes variations des caractéristiques des éjaculats, d'une part en fonction de l'âge des coqs, d'autre part en fonction de la fréquence des collectes.

I. Sur le premier point, le fait qui nous semble le plus notable est une augmentation progressive de la proportion de spermatozoïdes mobiles en fonction de l'áge chez les jeunes coqs. Ceci explique, au moins en partie, pourquoi la fécondance des premiers éjaculats produits est faible chez le Coq (SAEKI, I963). Mais nous ignorons l'origine de cette faible motilité initiale des spermatozoïdes car elle peut être liée soit à un fonctionnement déficient de la spermatogenèse, soit à un défaut de maturation dans les voies déférentes.

Il en résulte qu'on ne peut définir la maturité sexuelle du Coq par l'apparition des premiers spermatozoïdes puisque ceux-ci ont une fécondance subnormale.

Pour notre part, nous estimons que la maturité sexuelle est atteinte quand le poids testiculaire, la quantité de spermatozoides produits et leur fécondance ont atteint leur valeur maximum. 
2. Sur le deuxième point, il a été plusieurs fois observé que le nombre de spermatozoïdes par éjaculat diminue quand la fréquence des collectes augmente. Mais le nombre de spermatozoïdes récoltés par unité de temps (i.e. par jour ou par semaine) est, par contre, d'autant plus élevé que la fréquence des collectes est plus grande (SWiERSTRA et S'TRAin, I964; de REviERs, I972 $b$ ).

Fait nouveau, nous observons que la proportion de spermatozoïdes mobiles augmente fortement lors du passage de 2 collectes par semaine à I collecte par jour. Il faut rapprocher ce fait d'un résultat de PETITJEAN (I970) montrant que la fécondance des spermatozoïdes de Coq est plus élevée quand 1'intervalle entre 2 collectes successives n'est que d'une demi-journée au lieu de 3 jours. Cette augmentation de motilité et de fécondance, lors de collectes fréquentes est probablement en rapport avec la durée de séjour des spermatozoïdes dans les voies déférentes. En d'autres termes, il y aurait peut-être un certain "vieillissement " des spermatozoïdes dans les voies déférentes quand la fréquence des collectes est trop peu élevée.

Nous sommes donc amenés à penser que les collectes de spermatozoïdes effectuées en vue de l'insémination artificielle doivent être relativement fréquentes. Dans l'état actuel de nos connaissances, un intervalle de 24 heures entre 2 collectes successives nous paraît satisfaisant aussi bien pour le nombre des spermatozoïdes récoltés que pour leur qualité.

3. Par ailleurs, nos résultats montrent que le gène $\mathrm{dw}$ à l'état homozygote déprime la production spermatique au plan quantitatif, mais la stimule, au contraire, au plan qualitatif, au moins pour la motilité.

A $u$ plan quantitatif, l'importante différence qui se manifeste entre les coqs $d w d w$ et les coqs Dwdw pour les nombres testiculaires ne se répercute que d'une manière très atténuée sur le nombre de spermatozoïdes récoltés dans les éjaculats. A quoi tient cette atténuation? L'une de ses causes est probablement à rechercher dans la fréquence des collectes qui a peut-être été trop faible pour que la différence de production testiculaire se manifeste de manière importante dans les éjaculats. Le fait d'avoir interrompu les collectes le dimanche peut avoir eu des conséquences : un travail en cours tend à nous le confirmer.

Au plan qualitatif, les coqs dwdw produisent les spermatozoïdes ayant une motilité supérieure à celle qui est observée chez les coqs $D w d w$. Nous avons observé par ailleurs que le gène $d w$ à l'état homozygote est capable d'améliorer la fécondance des spermatozoïdes même chez des coqs $R R(R=$ gène de crête en rose, provoquant la subfertilité des coqs) (PETITJEAN, résultats non publiés). Ainsi, dw et $R$ paraissent antagonistes par leurs effets sur la motilité des spermatozoïdes, actions que nous sommes actuellement incapables d'expliquer.

La différence de développement corporel que l'on observe entre les coqs $D w d w$ et $d w d w$ ne nous semble pas pouvoir expliquer la différence observée pour le poids testiculaire, car il est réduit dans une proportion bien plus grande que le poids corporel chez les coqs $d w d w$ : en moyenne le rapport $\frac{\text { poids testiculaire }}{\text { poids corporel }}$ est de o,49 p. roo chez les coqs $d w d w$ au lieu de o,70 p. Ioo chez les coqs $D w d w$; la différence observée entre ces deux rapports étant hautement significative. Nous nous demandons alors à quel niveau agit le gène $d w^{\prime}$. Est-ce sur les testicules eux-mêmes ou sur le complexe hypothalamo-hypophysaire? 


\section{SUMMARY}

\section{INFLUENCE OF THE $d w$ GENE ON THE SPERM PRODUCTION AND THE SPERM OUTPUT IN THE GROWING COCKEREL}

The number of ejaculated spermatozoa is lesser among $d w d w$ cockerels than among $D w d w$ cockerels when they are 14 to 28 weeks old. In adults, this difference is only 25 p. roo. Meanwhile, testicular weight is much lighter in $d w d w$ cockerels ( $10.8 \pm 0.5 \mathrm{~g}$ ) than in Dwdw cockerels $(25.2 \pm$ I.I g); We observed that the $d w$ locus seems to depress testicular weight more than body weight. The motility of the ejaculated spermatozoa is higher among $d w d w$ cockerels than among Dwdw cockerels.

In both types, the motility of the first ejaculated spermatozoa appeared very low ; it increase with age from the I 4 th to the I 8 th week. Furthermore the motility of cock's spermatozoa sharply increases when semen collections become more frequent.

\section{RÉFÉRENCES BIBLIOGRAPHIQUES}

Burrows W. H., Quins J. P., I937. The collection of spermatozoa from the domestic fowl and turkey. Poult. Sci., 16, 19-24.

Hutr F. B., 1949. Genetics of the fowl. Mc Graw Hill Books, New York, 263-264.

Ortavant R., I958. "Le cycle spermatogénétique chez le Bélier ". Thèse D. Sc., Paris, r 27 p. ; éd. r959. Ann. Zootech., I, I83-244 et 27I-322.

Petitjean M., I97o. Résultats expérimentaux sur la subfertilité liée à la crête rosacée chez le Coq. $X I V^{\mathrm{e}}$ Congrès Mondial d'Aviculture, Madrid, 6-I2, septembre 1970.

Petitjean M.-J., Reviers M. de, x973. Effets du gène de nanisme $d w$ sur la maturité sexuelle des coqs. 4th Poult. Conf., London, 569-575.

Reviers M. de, I972a. Évaluation des réserves spermatiques dans les voies déférentes du Coq. $A n n$. Biol. anim. Bioch. Biophys., 12, 5-Ir.

Reviers M. de, I972 b. Évaluation de la production de spermatozoïdes chez le Coq. Ann. Biol. anim. Bioch. Biophys., 12, I-I8.

Ricard F. H., I970. Étude d'un gène de nanisme lié au sexe chez la Poule. V. Observations sur la crois. sance et les caractéristiques de carcasse du jeune poulet. Ann. Génét. Sél. anim., 2, r9-3r.

SAEKI Y. I963. Fertilizing ability of cock spermatozoa first ejaculated and changes in semen quality with age of the cock. Jap. J. Zoot. Sci., 34, I I-I25.

Swierstra E. E., Strain J. H., I964. Sperm output of White Leghorn roosters ejaculated daily or every other day for eight weeks. Poult. Sci., 43, I368. 